\title{
Spitzer view on the downsizing scenario of galaxy formation and the role of AGN
}

\author{
Pablo G. Pérez-González ${ }^{1}$, Almudena Alonso-Herrero ${ }^{2}$, Jennifer Donley ${ }^{3}$, George \\ Rieke $^{3}$, Guillermo Barro ${ }^{1}$, Jesús Gallego ${ }^{1}$, and Jaime Zamorano ${ }^{1}$
}

\begin{abstract}
We present the latest results of the Spitzer Cosmological Surveys concerning the characterization of the evolution of galaxies in the last $12 \mathrm{Gyr}$ (from $\mathrm{z}=4$ ). We have analyzed the stellar mass function up to $\mathrm{z}=4$ using a sample of more the 28,000 galaxies selected in the rest-frame near-infrared with Spitzer/IRAC. Our results confirm and quantify the "downsizing" scenario of galaxy formation. Based on the study of the specific SFRs of X-ray emitters, we discuss the role of AGN in the evolution of galaxies, arguing against the link between nuclear activity and the quenching of the star formation in massive galaxies at $\mathrm{z}<1.4$.
\end{abstract}

\section{Introduction}

One of the most interesting results in Extragalactic Astronomy in the last decade is the discovery of a numerous population of massive galaxies $\left(\mathscr{M}>10^{11} \mathscr{M}_{\odot}\right.$, similar to a local elliptical) at high redshift (Yan et al. 1999; Franx et al. 2003), being some of them already evolving passively (Daddi et al. 2004). Even more puzzlingly, these galaxies present very small sizes, and thus large mass densities (e.g., Daddi et al. 2005; Trujillo et al. 2007) comparable to the density of a globular cluster (Buitrago et al. 2008). This finding is extremely challenging for models of galaxy formation, based on the hierarchical $\Lambda$ CDM paradigm (e.g., Baugh et al. 1996; Cole et al. 2000), which have had to introduce in their formulations early massive star formation (with a cosmic SFR density staying roughly constant at $\mathrm{z}=1-3$; Madau et al. 1996; Pérez-González et al. 2005, and references therein; Hopkins \& Beacom 2006) and quenching mechanisms, such as AGN feedback, at high redshift to try to reconcile with the observations (e.g., de Lucia et al. 2006; Croton et al. 2006).

In the local universe, massive quiescent early-type galaxies are 3 times more numerous than late-type galaxies above a given mass threshold (Baldry et al. 2004). A color-magnitude diagram clearly separates the red quiescent galaxies (populating the so-called "red sequence") from the blue star forming galaxies (e.g., Kauffmann et al. 2003). The majority of these massive galaxies was already in place at $\mathrm{z} \sim 1$ (Bell et al. 2004; Bundy et al. 2007; Faber et al. 2007; Conselice et al. 2007). In addition, several works have found compelling evidence of a high formation redshift ( $\mathrm{z} \sim 4$ ) for the most massive galaxies (Heavens et al. 2004; Juneau et al. 2005;

${ }^{1}$ Universidad Complutense de Madrid, Spain; ${ }^{2}$ IEM/CSIC, Spain; ${ }^{3}$ University of Arizona, USA. 
Pérez-González et al. 2005; Cimatti et al. 2006), a scenario known as downsizing (Cowie et al. 1996). Therefore, the key epoch for the study of the formation of the red sequence is $1<\mathrm{z}<3$ (Fontana et al. 2004; Abraham et al. 2007; Arnouts et al. 2007). From all these results we can infer that, in order to understand galaxy evolution, it is crucial to construct and analyze in detail statistically meaningful samples of galaxies selected by their stellar mass at $\mathrm{z}=0-4$. And among them, some of the most interesting systems are those which have completely stopped forming stars (totally quiescent), as they should shed light on the quenching mechanisms of galaxy formation.

In this summary of our oral contribution to the VIII Spanish Astronomical Society Meeting, we summarize our efforts to study the mass assembly of galaxies in the last 12 Gyr $(\mathrm{z}=0-4)$ based on the characterization of a sample of galaxies selected by an observable close to the stellar mass: their rest-frame NIR emission. This selection has been feasible for the first time with the Cosmological Surveys carried out with Spitzer and its IRAC instrument, observing at 3.6-8.0 $\mu \mathrm{m}$. These data have been compiled by our group jointly with other public and proprietary ancillary observations in the UV/optical/NIR to study how fast and how much stellar mass has been assembled in galaxies from $\mathrm{z}=4$, study the relationship of the mass assembly with morphology (see Perez-Gonzalez et al. 2008), and test the AGN feedback quenching mechanism introduced by models to stop star formation in massive galaxies.

\section{The Rainbow Cosmological Surveys database at UCM}

The Rainbow Cosmological Surveys Database at UCM has been built by PGP-G, with the help of several people in the UCM Star Formation Group (SFG), and in the MIPS Instrument Team. Some of the main contributors are: Guillermo Barro, Jesús Gallego, George Rieke, Jaime Zamorano, Víctor Villar, Sergio Pascual, Armando Gil de Paz, Jennifer Donley, Eiichi Egami, Almudena Alonso-Herrero...

The Rainbow Cosmological Surveys Database includes the following fields:

-Chandra Deep field South (CDFS). We have complete catalogs for a $225 \mathrm{arcmin}^{2}$ region centered on $\alpha(\mathrm{J} 2000.0)=53^{\circ} .115 \delta(\mathrm{J} 2000.0)=-27^{\circ} .805$ covering the whole IRAC GOODS-S field.

-Hubble Deep Field North (HDFN). We have complete catalogs for a 258 $\operatorname{arcmin}^{2}$ region centered on $\alpha(\mathrm{J} 2000.0)=189^{\circ} .235 \delta(\mathrm{J} 2000.0)=62^{\circ} .235$ covering the whole IRAC GOODS-N field.

-Lockman Hole Field (LHF). We have complete catalogs for a $182 \mathrm{arcmin}^{2}$ region centered on $\alpha(\mathrm{J} 2000.0)=162^{\circ} .195 \delta(\mathrm{J} 2000.0)=57^{\circ} .485$.

-Extended Groth Strip (EGS). We have complete catalogs for a $850 \mathrm{arcmin}^{2}$ region centered on $\alpha(\mathrm{J} 2000.0)=214^{\circ} .800 \delta(\mathrm{J} 2000.0)=52^{\circ} .810$, covering the whole IRAC EGS Survey.

-Subaru XMM Deep Field (SXDS). We have complete catalogs for $2000 \mathrm{arcmin}^{2}$ region centered on $\alpha(\mathrm{J} 2000.0)=34^{\circ} .400 \delta(\mathrm{J} 2000.0)=-5^{\circ} .100$ covering the whole UKIDSS UDS and the Spitzer Legacy (SpUDS) area. 
To show what is available in the Rainbow database, we have released a limited part of the CDFS data and results. This is accessible from the Rainbow_Navigator web interface: http://guaix.fis.ucm.es/ pgperez/Proyectos/ucmcsdatabase.en.html.

This public release includes the SEDs, spectroscopic redshifts, photometric redshifts, stellar masses, and SFR estimates for all the IRAC $3.6 \mu \mathrm{m}$ selected sources and all the $I$-band selected sources in a $2^{\prime} \times 2^{\prime}$ region in the center of the GOODS-S field. The central coordinates of the publicly available region are $\alpha(\mathrm{J} 2000.0)=53^{\circ} .115 \delta(\mathrm{J} 2000.0)=-27^{\circ} .805$. We refer the reader to the papers PérezGonzález et al. (2005) and Pérez-González et al. (2008) for a description of the data compilation and the derivation of interesting parameters.

\section{The Stellar Mass Function up to $z=4$}

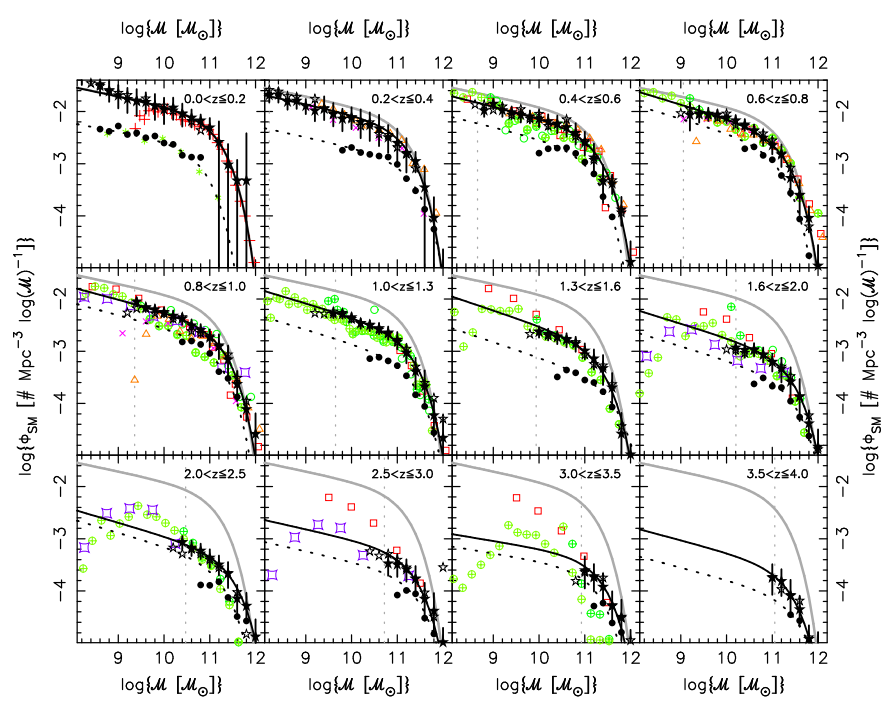

Fig. 1 Stellar mass functions in 12 redshift intervals from $z=0$ to $z=4$. Our estimations at each redshift interval are plotted with black filled stars and errors for an IRAC selected sample, and with open black stars for an $I$-band selected sample (errors for this sample are not plotted for clarity). Filled circles show the SMF for galaxies detected by MIPS at $24 \mu \mathrm{m}$. Color points show other estimates found in the literature based on samples selected in a variety of ways. Extracted from Pérez-González et al. (2008).

Figure 1 shows the evolution of the stellar mass function (SMF) from $\mathrm{z}=0$ to $\mathrm{z}=4$, according to our analysis of a MIR-selected sample. Our IRAC-based selection at $3-5 \mu \mathrm{m}$ is the closest to a stellar mass selected sample up to the highest redshifts, in comparison with other samples selected in the optical or NIR (color points in Figure 1), which probe the rest-frame UV (where the emission is normally dominated by young stars which do not typically contribute much to the global stel- 
lar mass) as we move to higher redshifts. Our results show that the local density of galaxies (shown with a gray line in all panels) with masses $\mathscr{M}>10^{12} \mathscr{M} \odot$ was already reached by the $\mathrm{SMF}$ at $\mathrm{z}=2.5-3.0$, i.e., the most massive galaxies were already in place at that redshift (approximately $11 \mathrm{Gyr}$ ago). The mass assembly of galaxies shifts to smaller masses as we move to lower redshifts. By $\mathrm{z} \sim 1$, the SMF has reached the local density for galaxies with $\mathscr{M}>10^{11.8} \mathscr{M}_{\odot}$. At $\mathrm{z}<1$, the star formation in the Universe occurs mainly in galaxies with $\mathscr{M}<10^{11.5} \mathscr{M} \odot$. The filled circles in each panel of Figure 1 show the SMF for galaxies detected by MIPS at $24 \mu \mathrm{m}$, i.e., galaxies which are currently forming stars. Comparing these SMFs with those of the global population of galaxies, we clearly see that the density of galaxies detected by MIPS for a given stellar mass gets closer to the global density as we move to higher redshifts. This means that not only the most massive galaxies formed earlier in the history of the Universe, but also with more intense bursts (with SFRs of several hundred of $\mathscr{M}_{\odot} / \mathrm{yr}$ ) and, consequently, more rapidly. These are two clear evidences of downsizing. We refer the reader to Pérez-González et al. (2008) for further discussion on this topic, and for more details on our quantification of the downsizing scenario of galaxy formation.

\section{Downsizing in depth: AGN as a quenching mechanism}

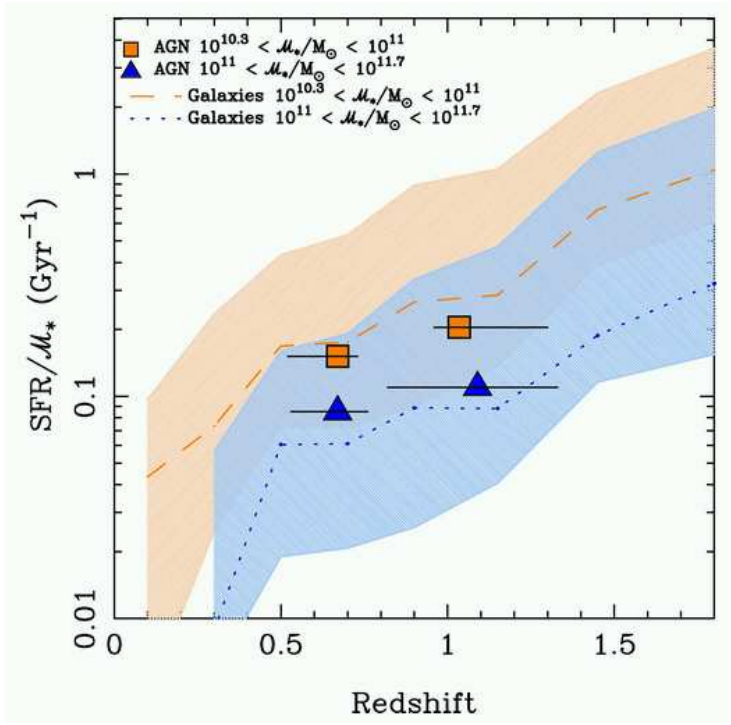

Fig. 2 Redshift evolution of the specific SFRs for IRAC-selected galaxies (from Pérez-González et al. 2008, color lines and shaded regions) and for the sample of X-ray emitting AGNs in the CDFS. The median specific SFRs for AGNs are shown by the filled squares and triangles plotted at the median redshift. The horizontal bars represent the ranges of redshifts. Figure extracted from Alonso-Herrero et al. (2008). 
If we accept the downsizing theory, one can question why the most massive galaxies started forming soon in the history of the Universe and why they stopped forming stars at some time. Recent models based on a $\Lambda \mathrm{CDM}$ cosmology succeed in predicting the early formation of massive galaxies by introducing very large dust extinctions, non-standard Initial Mass Functions (IMFs), and/or suppression of the star formation due to the quenching of cooling flows due to supernovae or Active Galactic Nuclei (e.g., Cole et al. 2000; Granato et al. 2004; Baugh et al. 2005; Nagamine et al. 2005; Croton et al. 2006; Bower et al. 2006).

From the observational point of view, it seems that the AGN possibility is favored. For example, Sánchez et al. (2004) and Nandra et al. (2007) found that X-ray emitting AGNs tend to concentrate in a color-magnitude diagram in a region connecting the red sequence and the blue cloud, the so-called green valley. This zone would be transitory for massive galaxies which are stopping their star formation and moving to the quiescent state towards the red sequence. Bundy et al. (2008) also found evidence for AGN being responsible for the star formation quenching: they deduced that the AGN "trigger" rate matches the star formation quenching rate, suggesting a link between these phenomena.

Concerning the possibility of a change in the IMF, we refer the reader to several recent papers, all of them pointing out to an evolution towards top-heavy IMFs at higher redshifts: Pérez-González et al. (2008), van Dokkum (2008), Davé (2008), among others.

In Alonso-Herrero et al. (2008), we analyzed the SFRs and specific SFRs of the hosts of X-ray bright AGN. The main results of this paper are summarized in their Figure 7, which we replicate here. Alonso-Herrero et al. (2008) demonstrate that the star formation in X-ray detected AGN is not suppressed in comparison with the global population of galaxies with a similar stellar mass (in the interval $\left.10.3<\log \left(\mathscr{M} / \mathscr{M}_{\odot}\right)<11.7\right)$ for galaxies at $0.4<\mathrm{z}<1.3$. This seems to rule out AGN as the dominant detonator in quenching the star formation in massive galaxies at intermediate redshift. However, this interpretation may be affected by the bias imposed by the X-ray selection of the AGN studied in Alonso-Herrero et al. (2008). Indeed, the X-ray bright may be just a phase of AGN (maybe linked to mergers; e.g, Hopkins et al. 2008), and it may happen early in their evolution so the star formation is not suppressed yet. Other phases may follow, such as a QSO phase or/and a obscured AGN phase, which may be more directly linked to the star formation suppression.

\section{References}

Abraham, R. G. et al. 2007, ApJ, 669, 184

Alonso-Herrero, A., Pérez-González, P. G., Rieke, G. H., Alexander, D. M., Rigby, J. R., Papovich, C., Donley, J. L., \& Rigopoulou, D. 2008, ApJ, 677, 127

Arnouts, S. et al. 2007, A\&A, 476, 137

Baldry, I. K., Glazebrook, K., Brinkmann, J., Ivezić, Ž., Lupton, R. H., Nichol, R. C., \& Szalay, A. S. 2004, ApJ, 600, 681

Baugh, C. M., Cole, S., \& Frenk, C. S. 1996, MNRAS, 283, 1361 
Baugh, C. M., Lacey, C. G., Frenk, C. S., Granato, G. L., Silva, L., Bressan, A., Benson, A. J., \& Cole, S. 2005, MNRAS, 356, 1191

Bell, E. F. et al. 2004, ApJ, 608, 752

Bower, R. G., Benson, A. J., Malbon, R., Helly, J. C., Frenk, C. S., Baugh, C. M., Cole, S., \& Lacey, C. G. 2006, MNRAS, 370, 645

Buitrago, F., Trujillo, I., Conselice, C. J., Bouwens, R. J., Dickinson, M., \& Yan, H. 2008, astro-ph/0807.4141

Bundy, K. et al. 2008, ApJ, 681, 931

Bundy, K., Treu, T., \& Ellis, R. S. 2007, ApJL, 665, L5

Cimatti, A., Daddi, E., \& Renzini, A. 2006, A\&A, 453, L29

Cole, S., Lacey, C. G., Baugh, C. M., \& Frenk, C. S. 2000, MNRAS, 319, 168

Conselice, C. J. et al. 2007, ApJL, 660, L55

Cowie, L. L., Songaila, A., Hu, E. M., \& Cohen, J. G. 1996, AJ, 112, 839

Croton, D. J. et al. 2006, MNRAS, 365, 11

Daddi, E., Cimatti, A., Renzini, A., Fontana, A., Mignoli, M., Pozzetti, L., Tozzi, P., \& Zamorani, G. 2004, ApJ, 617, 746

Daddi, E. et al. 2005, ApJ, 626, 680

Davé, R. 2008, MNRAS, 385, 147

de Lucia, G., Springel, V., White, S. D. M., Croton, D., \& Kauffmann, G. 2006, MNRAS, 366, 499

Faber, S. M. et al. 2007, ApJ, 665, 265

Fontana, A. et al. 2004, A\&A, 424, 23

Franx, M. et al. 2003, ApJL, 587, L79

Granato, G. L., De Zotti, G., Silva, L., Bressan, A., \& Danese, L. 2004, ApJ, 600, 580

Heavens, A., Panter, B., Jimenez, R., \& Dunlop, J. 2004, Nature, 428, 625

Hopkins, A. M., \& Beacom, J. F. 2006, ApJ, 651, 142

Hopkins, P. F., Cox, T. J., Kereš, D., \& Hernquist, L. 2008, ApJS, 175, 390

Juneau, S. et al. 2005, ApJL, 619, L135

Kauffmann, G. et al. 2003, MNRAS, 341, 54

Madau, P., Ferguson, H. C., Dickinson, M. E., Giavalisco, M., Steidel, C. C., \& Fruchter, A. 1996, MNRAS, 283, 1388

Nagamine, K., Cen, R., Hernquist, L., Ostriker, J. P., \& Springel, V. 2005, ApJ, 627, 608

Nandra, K. et al. 2007, ApJL, 660, L11

Pérez-González, P. G. et al. 2005, ApJ, 630, 82

-. 2008, ApJ, 675, 234

Perez-Gonzalez, P. G., Trujillo, I., Barro, G., Gallego, J., Zamorano, J., \& Conselice, C. J. 2008, astro-ph/0807.1069

Sánchez, S. F. et al. 2004, ApJ, 614, 586

Trujillo, I., Conselice, C. J., Bundy, K., Cooper, M. C., Eisenhardt, P., \& Ellis, R. S. 2007, MNRAS, 382, 109

van Dokkum, P. G. 2008, ApJ, 674, 29

Yan, L., McCarthy, P. J., Freudling, W., Teplitz, H. I., Malumuth, E. M., Weymann, R. J., \& Malkan, M. A. 1999, ApJL, 519, L47 